\title{
SPIN EFFECTS IN NUCLEON-NUCLEON ELASTIC SCATTERING
}

Kent M. Terwilliger

The University of Michigan, Ann Arbor, Michigan 48109

Three papers involving spin effects in high momentum transfer nucleon-nucleon elestic scattering are briefly summarized.

POLARIZATION IN LARGE ANGLE PROTON-NEUTRON ELASTIC SCATTERING*

Y. Makdisi, M.L. Marshak, B. Mossberg, E.A. Peterson, K. Ruddick School of Physics and Astronomy, University of Minnesota Minneapolis, Minnesota 55455

J.B. Roberts

Physics Department and T.W. Bonner Nuclear Laboratories Rice University, Houston, Texas 77001

R.D. Klem

Argonne National Laboratory, Argonne, IL 60439

The authors have measured the large angle polarization asymmetry $A$ in the proton-neutron elastic scattering at 2,3 , and $6 \mathrm{GeV} / \mathrm{c}$ using the polarized proton beam at the Argonne ZGS and a liquid deuterium target. These measurements, the first at high energy, show that $A$ is large (20-40\%) and negative at the larger angles, larger and opposite sign to PP scattering, and with no decrease with incident energy, unlike the earlier data at smaller angles. At $90^{\circ} \mathrm{cm}$, where A for pp is constrained to be zero because of particle identity, the np asymmetry is increaslng with energy, reaching approximately -.3 at $6 \mathrm{GeV} / \mathrm{c}$, in conflict with the basic constituent interchange model which predicts $A$ for np scattering to be 0 at $90^{\circ} \mathrm{cM}$.

tWork supported in part by the U.S. Department of Energy and by the Graduate School of the University of Minnesota.

SPIN-SPIN FORCES IN $6 \mathrm{GEV/C} \mathrm{NEUTRON-PROTON} \mathrm{ELASTIC} \mathrm{SCATTERING}$

ENERGY DEPENDENCE OF SPIN-SPIN EFFECTS IN P-P ELASTIC SCATTERING AT $90^{\circ} \mathrm{CM}$

D.G. Crabb, R.C. Fernow, P.H. Hansen, A.D. Krisch, B. Sandler, T. Shima and K.M. Terwilliger

Randall Laboratory of Physics, The University of Michigan Ann Arbor, Michigan 48109

J.R. O'Fallon

Argonne Universities Association, Argonne, 111 inois 60439

0094-243X/81/680054-02\$1.50 Copyright 1981 American Institute of Physics 
E.A. Crosbie, L.G. Ratner, P.F. Schultz, G.H. Thomas Argonne National Laboratory, Argonne, 111 inois 60439

N.L. Karmarkar

University of Kiel, Kiel, Germany

S.L. Linn and A. Perlmutter

Department of Physics and Center for Theoretical Studies

The University of Miami, Coral Gables, Florida 33124

A. Lin

Abadan Institute of Technology, Abadan, Iran

A.J. Salthouse

Bell Laboratories, Murray Hill, New Jersey 07974

P. Kyberd

Nuclear Physics Laboratory

Oxford University, Oxford, England

In the two-spin experiment $n \uparrow+p \uparrow \rightarrow n+p$ we measured do/dt at $P^{2}=0.8$ and $1.0(\mathrm{GeV} / \mathrm{c})^{2}$ at $6 \mathrm{GeV} / \mathrm{c}$. We used the $6 \mathrm{GeV} / \mathrm{c} 53 \%$ polarized neutrons from the $12 \mathrm{GeV} / \mathrm{c}$ polarized deuteron beam at the Argonne ZGS, and scattered them from our $75 \%$ polarized proton target. Both spins were oriented perpendicular to the scattering plane. We found interesting spin-spin effects in $n-p$ elastic scattering: $A_{n n}=-.17 \pm .05$ at $P^{2}=0.8$, and $A_{n n}=-.19 \pm .05$ at $P^{2}=1.0$ $(\mathrm{GeV} / \mathrm{c})^{2}$. These values are larger in magnitude and opposite in sign from $A_{n n}$ in $P p$ elastic scattering at $6 \mathrm{GeV} / c$ at the same $P_{1}^{2}$. The basic consitituent interchange model predicts the np $A_{n n}$ to be -.44 .

In the two-spin experiment $p \uparrow+p \uparrow \rightarrow p+p$ the energy dependence of the spin-parallel and spin-antiparallel cross-sections at $90^{\circ} \mathrm{CM}$, with spins normal to the scattering plane, was measured for beam momenta between $6 \mathrm{GeV} / \mathrm{c}$ and $12.75 \mathrm{GeV} / \mathrm{c}$. The ratio (do/dt) parallel: (do/dt) antiparallel is about 1.2 up to $8 \mathrm{GeV} / \mathrm{c}$ and then increases rapidly to gave of almost 4 near $11 \mathrm{GeV} / \mathrm{c}$ ( $A_{n n}$ goes from approximately .1 to .6 ). The highest momenta points suggest that the ratio may reach a limiting value of about 4 . When plotted against $\mathrm{P}^{2}$ this rapid increase in cross section ratio closely matches that observed earlier at the fixed laboratory momentum of $11.75 \mathrm{GeV} / \mathrm{c}$, where the scattering angle was varied. This close correspondence suggests that the pure spin cross sections may be mainly dependent on $P_{1}$ ? in the hard scattering region. The data are in strong disagreement with the basic constituent interchange model, which predicts a cross-section ratio of 2 .

This work was supported by the U.S. Department of Energy 